\title{
A simple method to control glycolytic flux for the design of an optimal cell factory
}

\author{
Jae Hyung Lim ${ }^{1}$ and Gyoo Yeol Jung ${ }^{1,2^{*}}$ (D)
}

\begin{abstract}
Background: A microbial cell factory with high yield and productivity are prerequisites for an economically feasible bio-based chemical industry. However, cell factories that show a kinetic imbalance between glycolysis and product formation pathways are not optimal. Glycolysis activity is highly robust for survival in nature, but is not optimized for chemical production.

Results: Here, we propose a novel approach to balance glycolytic activity with the product formation capacity by precisely controlling expression level of pts $G$ (encoded glucose transporter) through UTR engineering. For various heterologous pathways with different maximum production rates, e.g., $n$-butanol, butyrate, and 2,3-butanediol, glycolytic fluxes could be successfully modulated to maximize yield and productivity, while minimizing by-product formation in Escherichia coli.

Conclusions: These results support the application of this simple method to explore the maximum yield and productivity when designing optimal cell factories for value-added products in the fields of metabolic engineering and synthetic biology.
\end{abstract}

Keywords: Glycolytic flux, n-Butanol, 2,3-Butanediol, Butyrate, UTR engineering, PTS

\section{Background}

Optimal microbial cell factories are essential to develop economically feasible production processes for various value-added chemicals from renewable biomass at an industrial scale [1]. Therefore, the design of cell factories in the fields of metabolic engineering and synthetic biology aims to maximize cellular performance in terms of yield and productivity. This optimization is particularly important for high-volume (and low-value) bulk chemicals and biofuels [2], e.g., $n$-butanol (an alternative to gasoline) [3], butyrate (chemical feedstock for plastics) [4], and 2,3-butanediol (for rubbers) [5].

In general, cell factories can be simplified into two parts: a carbon utilization pathway, such as glycolysis, and a product formation pathway (Fig. 1). Traditionally, research in this field has focused on the product-forming

\footnotetext{
*Correspondence: gyjung@postech.ac.kr

${ }^{1}$ Department of Chemical Engineering, Pohang University of Science and Technology, 77 Cheongam-Ro, Nam-Gu, Pohang, Gyeongbuk 37673 , Korea

Full list of author information is available at the end of the article
}

pathways of interest. Yield can be enhanced by rerouting the carbon flux toward the target product by eliminating endogenous side reactions, and productivity can be improved by increasing the catalytic activity of kinetic bottlenecks in the product formation pathway [6-8]. However, we additionally speculated that the kinetic imbalance between glycolysis and product formation pathways should be considered in the design principle for optimal cell factories to maximize yield and productivity. When the maximum catalytic activity of the engineered pathway, i.e., the capacity of the product formation pathway, is lower than the glycolytic activity, additional carbon inputs can be wasted as by-products and the yield is consequently reduced (Fig. 1, Product A). In contrast, if the product formation capacity exceeds the glycolytic flux, glycolytic activity can be regarded as the rate-limiting step and increases in activity are necessary to improve productivity (Fig. 1, Product B). Pyruvate, for example, is a critical intermediate between sugar uptake and product formation as a final metabolite of glycolysis in almost all organisms [9]. Excess pyruvate, i.e., quantities that 


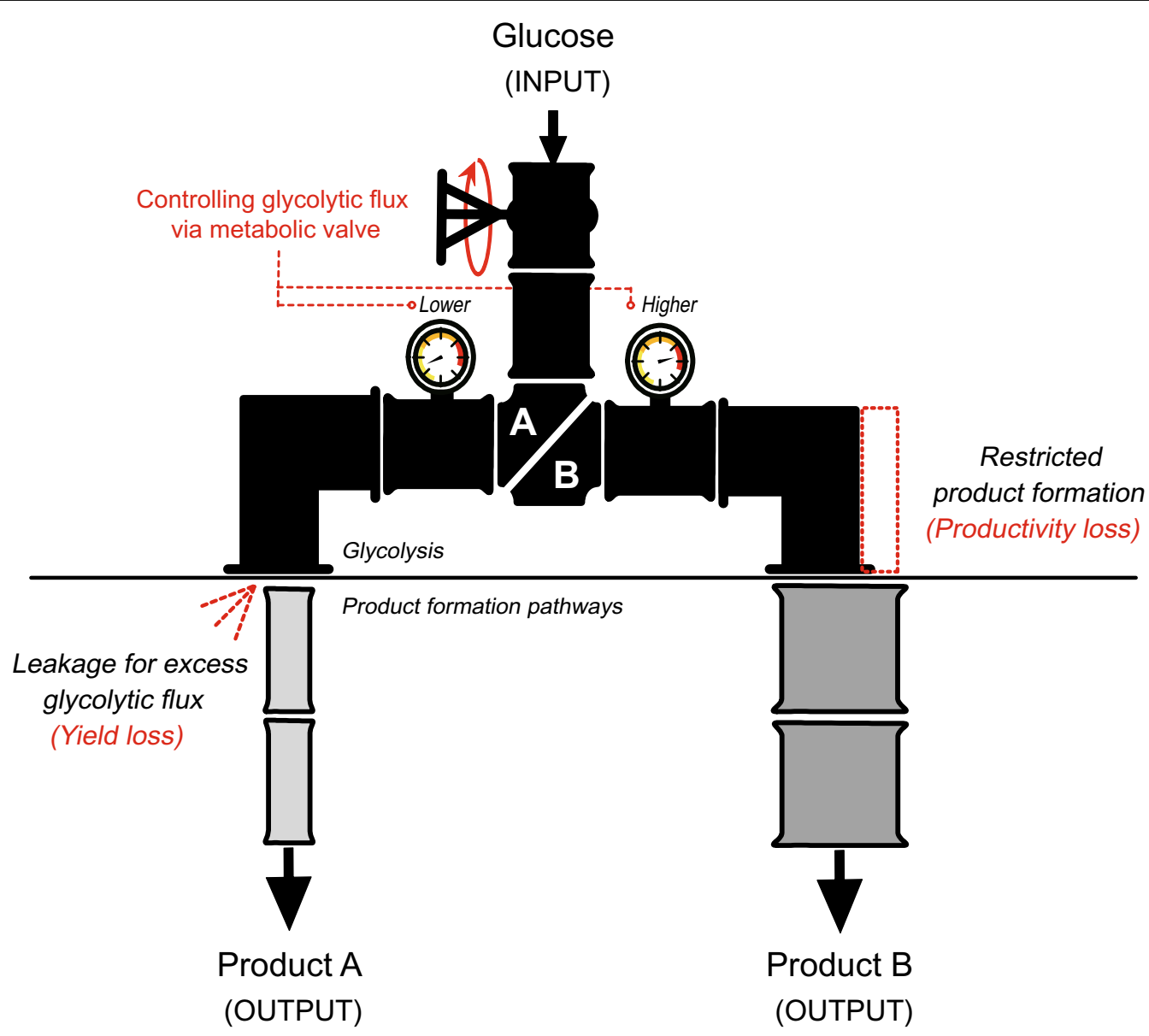

Fig. 1 Schematic diagram describing the discrepancy in efficiency between glycolysis and the product-forming pathway and the concept of a

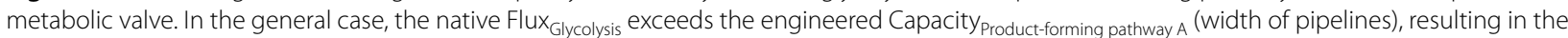

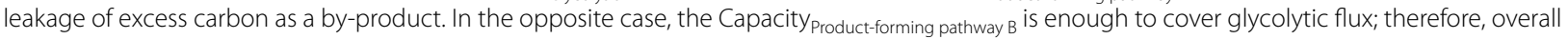

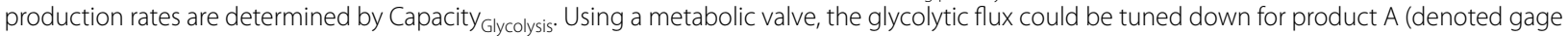
as 'lower') or amplified for product B (denoted gage as 'higher')

exceed the requirement for product formation, is inevitably secreted from the cell $[10,11]$, causing a substantial reduction in yield, whereas a lack of pyruvate limits the product formation rate, i.e., reduces productivity (Fig. 1). Taken together, a balance between glycolysis and product formation is required to construct a microbial cell factory with the maximum performance and this can be achieved via the precise control of glycolysis to maintain a balance with the capacity of the product formation pathway [12, 13].

However, methods to control the glycolytic flux are not well-studied owing to the robustness of native glycolytic activity, which is mediated by complex regulatory systems at many levels, including transcription, translation, and the allosteric control of enzymes [14]. Therefore, we focused on the carbon uptake system for the artificial control of carbon influx and simultaneously attempted to detour innate cellular regulatory mechanisms. There are several routes to start glycolysis in bacteria. For example, the phosphoenolpyruvate (PEP)-dependent sugar phosphotransferase system (PTS) that predominantly participates in both the transportation and phosphorylation of glucose. Alternatively, glucose can be internalized by a galactose transporter (GalP or MglABC) and subsequently phosphorylated by hexokinase to enter glycolysis. As such alternative pathways enable to decouple glucose transportation and PEP-dependent phosphorylation, and therefore the pathways were previously exploited to increase precursor availability, such as PEP and free glucose, for the production of aromatic amino acids $[15,16]$ and gluconic acid $[17,18]$, respectively. Strikingly, however, the PTS is the most efficient system in terms of energetic costs and kinetic parameters for glucose transportation among the routes [19]. The group translocation 
system is composed of non-sugar-specific soluble proteins: the phosphohistidine carrier protein $(\mathrm{HPr})$ and the Enzyme I (EI) component (encoded by pts $H$ and ptsI, respectively), the glucose-specific cytoplasmic enzyme EIIA (EIIA ${ }^{\text {Glc }}$, encoded by $\mathrm{crr}$ ), and the membranebound glucose-specific enzyme IICB (EIICB ${ }^{\text {Glc }}$, encoded by dicistronic $p t s G)[19,20]$. Moreover, PTS is primarily responsible for the control of glucose uptake in response to overflow glycolytic flux (for example, the accumulation of glucose-6-phosphate) via the post-transcriptional repression of $p t s G$ as the initial step in glucose import [21, 22]. Previous studies revealed that Escherichia coli small RNA SgrS is induced under glucose phosphate stress and it causes the translational repression and the RNaseE-dependent rapid degradation of the $p t s G$ mRNA [23] by binding to the $5^{\prime}$-end of mRNA [24, 25]. More recent work characterized a minimal base-pairing region between SgrS and ptsG mRNA that 14nt base-pairing region including Shine-Dalgarno (SD) sequence of the target mRNA is sufficient to inhibit ptsG translation (Fig. 2) [26].

In this study, we examined the potential use of $p t s G$ as a simple method to control the overall glycolytic flux simply by designing a synthetic $5^{\prime}$-untranslated region (UTR). UTR engineering is a suitable approach for controlling expression of target genes as well as for eliminating the unpredictable regulatory elements within the metabolic pathway [27]. Furthermore, we demonstrated the importance of rebalancing glycolytic flux depending on the efficiency of product formation pathways using recombinant $E$. coli strains producing $n$-butanol, butyrate, or 2,3-butanediol as model cell factories. Our approach enables the maximization of both yield and productivity in the construction of microbial cell factories by simply optimizing glycolytic flux; accordingly, it has broad applications for the cost-effective production of various chemicals and fuels.

\section{SgrS 3'-GGUUAUGAGUCAGUGUGU-ACUACG-UCC-5'

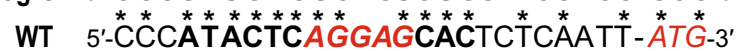

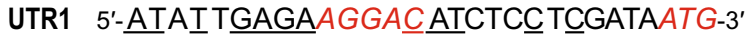 UTR2 5'-ATAT TGAGAAGGAG ATATCTCAATA-ATG-3' UTR3 5'-ATAITGAGAAGGAGTTATCTCGATA-ATG-3' UTR4 5'-ATAA CGAGTAGGAG TTTC TEGATA- - ATG-3'

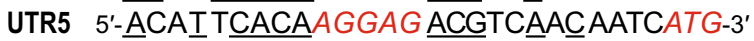

Fig. 2 Redesign of the 5'-UTR for ptsG based on base-pairs between SgrS and ptsG. The asterisks indicate the predicted base-pairing region of SgrS required for the translational repression of ptsG mRNA [24]. In particular, the minimal base-pairs for SgrS action for effective translational inhibition are shown in bold $[26,48]$. The italic letters represent the Shine-Dalgarno (SD) sequence and the initiation codon for pts $G$. The changed nucleotides with respect to the wild-type sequence of the ptsG UTR are underlined

\section{Methods}

\section{Reagents, bacterial strains, and plasmids}

A list of E. coli bacterial strains and plasmids used in this study is presented in Additional file 1: Table S1. Oligonucleotides used in this study were synthesized by Macrogen (Daejeon, Korea) and are listed in Additional file 1: Table S2. The rpsL-neo template DNA was obtained using the Counter-selection BAC Modification Kit (Gene Bridges, Heidelberg, Germany). Phusion DNA Polymerase and restriction endonuclease were supplied by New England Biolabs (Ipswich, MA, USA), and T4 DNA ligase was purchased from Takara Bio Inc. (Shiga, Japan). Genomic DNA and propagated plasmids were prepared using a GeneAll Exgene ${ }^{\mathrm{TM}}$ Cell SV Kit (GeneAll Biotechnology, Seoul, Korea) and an AccuPrep Nano-Plus Plasmid Mini Extraction Kit (Bioneer, Daejeon, Korea), respectively. Restriction enzyme-digested products were purified using a GeneAll Expin ${ }^{\mathrm{TM}}$ Gel SV Kit (GeneAll Biotechnology). All cell culture reagents were purchased from BD Biosciences (Sparks, MD, USA), and all other chemicals used in this study were purchased from Sigma (St. Louis, MO, USA), unless otherwise indicated.

Chromosomal modifications, including deletions and substitutions of the $5^{\prime}$-UTR of ptsG, were performed using the Red recombination system. Specifically, the knock-out mutant of ptsG was constructed using the Red recombination system with pKD46 and pCP20 [28, 29]. To increase the efficiency of homologous recombination, disruption cassettes with different priming sites (pFRT 4) were cloned and amplified using ptsG_del4_F and ptsG_del4_R, as described in our previous studies $[3,4]$. In addition, the replacement of the native UTR of ptsG was performed using the scar-less recombineering method [30] with Red recombination and the rpsL-neo counterselection system according to the manufacturer's instructions. For example, a mutation within the $r p s L$ gene that confers a streptomycin-resistant phenotype was introduced using rpsL-A128G-oligo. The resulting JHL163 ( $r p s L^{*}{ }_{\text {A128G }}$ ) strain was subjected to the insertion of a rpsL-neo cassette upstream of the ptsG structural gene, exhibiting recessive sensitivity to streptomycin in a merodiploid (JHL110). Finally, oligo recombination using [ptsG_UTR(1 to 5$)$ _oligo] that had distinctly redesigned $5^{\prime}$-UTR sequences based on UTR Designer (http://sbi. postech.ac.kr/utr_designer) [31] resulted in the ptsG UTR variants, UTR1, 2, 3, 4, and 5, without gaps (Fig. 2). The other strains were constructed in the same manner.

\section{Media and growth conditions}

Physiological analyses were conducted as follows: wildtype $E$. coli was aerobically cultivated using M9 medium (6.78 $\mathrm{g}$ of $\mathrm{Na}_{2} \mathrm{HPO}_{4}, 3 \mathrm{~g}$ of $\mathrm{KH}_{2} \mathrm{PO}_{4}, 1 \mathrm{~g}$ of $\mathrm{NH}_{4} \mathrm{Cl}$, $0.5 \mathrm{~g}$ of $\mathrm{NaCl}, 2 \mathrm{~mL}$ of $1 \mathrm{M} \mathrm{MgSO}_{4}$, and $0.1 \mathrm{~mL}$ of $1 \mathrm{M}$ 
$\mathrm{CaCl}_{2} / \mathrm{L}$ ) supplemented with $40 \mathrm{~g} / \mathrm{L}$ glucose [32]. Streptomycin $(25 \mu \mathrm{g} / \mathrm{mL})$ was used to determine the genotype of $\mathrm{rpsL}^{*}{ }_{\mathrm{A128G}}$. Overnight culture broths in LB medium were inoculated at approximately 1\% into M9 culture medium and cultivated until reaching an optical density at $600 \mathrm{~nm}\left(\mathrm{OD}_{600}\right)$ of $\sim 0.8$. The culture broths were inoculated at a final $\mathrm{OD}_{600}$ of 0.05 in $25 \mathrm{~mL}$ of $\mathrm{M} 9$ medium in a $300-\mathrm{mL}$ flask and incubated at $37{ }^{\circ} \mathrm{C}$ with shaking $(250 \mathrm{rpm})$. The production of $n$-butanol was assayed using Terrific Broth (TB; $12 \mathrm{~g}$ of tryptone, $24 \mathrm{~g}$ of yeast extract, $2.31 \mathrm{~g}$ of $\mathrm{KH}_{2} \mathrm{PO}_{4}, 12.54 \mathrm{~g}$ of $\mathrm{K}_{2} \mathrm{HPO}_{4}$, and $4 \mathrm{~mL}$ of glycerol per liter) supplemented with $25 \mathrm{~g} / \mathrm{L}$ glucose. Multiple plasmids were maintained using $25 \mu \mathrm{g} / \mathrm{mL}$ spectinomycin and $15 \mu \mathrm{g} / \mathrm{mL}$ kanamycin (pCDF-BuOH and pCOLA-F5). Rubber-sealed, 60-mL serum bottles were used for anaerobic cultures using an anaerobic chamber (Coy Laboratories, Ann Arbor, MI, USA). Overnight culture broths in LB medium were inoculated into $20 \mathrm{~mL}$ of fresh $\mathrm{TB}$ medium at a final $\mathrm{OD}_{600}$ of 0.05 and incubated anaerobically at $37^{\circ} \mathrm{C}$ in a rotary shaker $(250 \mathrm{rpm})$ [3]. The production of butyric acid was assayed using Terrific Broth (TB; $12 \mathrm{~g}$ of tryptone, $24 \mathrm{~g}$ of yeast extract, $2.31 \mathrm{~g}$ of $\mathrm{KH}_{2} \mathrm{PO}_{4}, 12.54 \mathrm{~g}$ of $\mathrm{K}_{2} \mathrm{HPO}_{4}$, excluding glycerol) supplemented with $10 \mathrm{~g} / \mathrm{L}$ glucose. The plasmid (pBASP) was maintained by including $34 \mu \mathrm{g} / \mathrm{mL}$ chloramphenicol. Rubber-sealed, 60-mL serum bottles were used for anaerobic cultures using an anaerobic chamber (Coy Laboratories). Overnight culture broths in LB medium were inoculated into $20 \mathrm{~mL}$ of fresh TB medium at a final $\mathrm{OD}_{600}$ of 0.05 and incubated anaerobically at $37^{\circ} \mathrm{C}$ in a rotary shaker (250 rpm) [4]. The production of 2,3-butanediol was tested using M9 medium $\left(6.78 \mathrm{~g}\right.$ of $\mathrm{Na}_{2} \mathrm{HPO}_{4}$, $3 \mathrm{~g}$ of $\mathrm{KH}_{2} \mathrm{PO}_{4}, 1 \mathrm{~g}$ of $\mathrm{NH}_{4} \mathrm{Cl}, 0.5 \mathrm{~g}$ of $\mathrm{NaCl}, 2 \mathrm{~mL}$ of $1 \mathrm{M}$ $\mathrm{MgSO}_{4}$, and $0.1 \mathrm{~mL}$ of $1 \mathrm{M} \mathrm{CaCl}_{2} / \mathrm{L}$ ) supplemented with $40 \mathrm{~g} / \mathrm{L}$ glucose and $5 \mathrm{~g} / \mathrm{L}$ yeast extract. The plasmid (pZSbudABC) was maintained by including $30 \mu \mathrm{g} / \mathrm{mL}$ kanamycin. Overnight culture broths in culture medium were inoculated into $100 \mathrm{~mL}$ of modified $\mathrm{M} 9$ medium at a final $\mathrm{OD}_{600}$ of 0.05 and incubated at $37{ }^{\circ} \mathrm{C}$ in a rotary shaker (180 rpm) under micro-aerobic condition. The anhydrotetracycline was added to a final concentration of $100 \mathrm{ng} /$ $\mathrm{mL}$ when the $\mathrm{OD}_{600}$ reached approximately 0.5 [5]. Theoretical yield was determined on the basis of pathway stoichiometry, e.g., $1 \mathrm{~mol}$ of $n$-butanol per $1 \mathrm{~mol}$ of glucose.

\section{Analytical methods}

The concentrations of glucose, organic acids, and alcohols were determined using high-performance liquid chromatography (UltiMate 3000 Analytical HPLC System; Dionex, Sunnyvale, CA, USA) with an Aminex HPX-87H Column (Bio-Rad Laboratories, Richmond, CA, USA) using $5 \mathrm{mM} \mathrm{H}_{2} \mathrm{SO}_{4}$ as the mobile phase. The 2,3-butanediol samples were analyzed at a flow rate of
$0.5 \mathrm{~mL} / \mathrm{min}$ at $65^{\circ} \mathrm{C}$ and otherwise a flow rate of $0.6 \mathrm{~mL} /$ min at $14{ }^{\circ} \mathrm{C}$ was used to quantify metabolites. The signal was monitored using a UV-Vis diode array detector (at $210 \mathrm{~nm}$ ) and a Shodex RI-101 detector (Shodex, Klokkerfaldet, Denmark).

\section{Glucose uptake rate}

The specific glucose uptake rate was determined as growth rate divided by biomass yield during exponential growth as previously described [33]. One $\mathrm{OD}_{600}$ unit corresponds to $0.25 \mathrm{~g}$ dry cell weight (DCW)/L [34]. Instead, glucose consumption rate, determined as the analytic data from HPLC during the initial exponential phase, was represented for the production systems as the components in the TB medium also contributed to biomass yield.

\section{Results}

Tuning glycolytic activity through UTR engineering of $p t s G$ We initially redesigned five $5^{\prime}$-UTR variants to control $p t s G$ activity as well as to deregulate translational repression [27] by the bacterial small RNA SgrS (which mediates phosphosugar stress responses) by modifying the minimal base-pairing region essential for SgrS action [26] (Fig. 2). As shown in Fig. 3b, seven strains with UTR variants, including positive $\left(\mathrm{UTR}_{\mathrm{WT}}\right)$ and negative $(\Delta p t s G)$ control strains, showed the various specific glucose uptake rates that were highly correlated with specific growth rates $\left(R^{2}=0.89\right)$ in the minimal medium. These results agree with previous continuous culture data indicating that the specific glucose uptake rate increases linearly as a function of the dilution or growth rate [33, 35]. Moreover, differences in the glucose consumption rate were also related to the accumulation of acetate $\left(R^{2}=0.88\right)$ and pyruvate $\left(R^{2}=0.77\right)$ (Fig. 3a, c, d). As the secretion of acetate and pyruvate is generally considered to result from a higher carbon flux than the flux through the TCA cycle, which is required for both biosynthesis and energy production (Fig. 3a) [10, 36], the accumulation of acetate and pyruvate as natural by-products in wild-type $E$. coli collectively represents glycolytic activity. Consequently, our results show that UTR engineering of ptsG could successfully modulate overall PTS activity (represented as the glucose uptake rate) and glycolytic flux.

Interestingly, the redesign of the upstream region of ptsG enabled a higher specific glucose uptake rate $(+20.8 \%)$, probably due to the deregulation of SgrS action, and subsequently led to a higher growth rate $(+7.3 \%)$ and higher accumulation of acetate $(+13.9 \%)$ and pyruvate $(+11.0 \%)$ than those of the parental strain (Fig. 3b-d). These results indicate that the glucose transporter (encoded by $p t s G$ ) can amplify glycolytic flux as a 
a

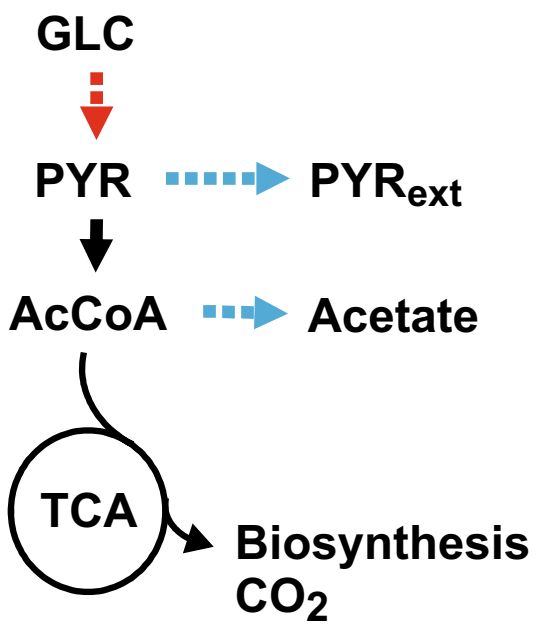

C

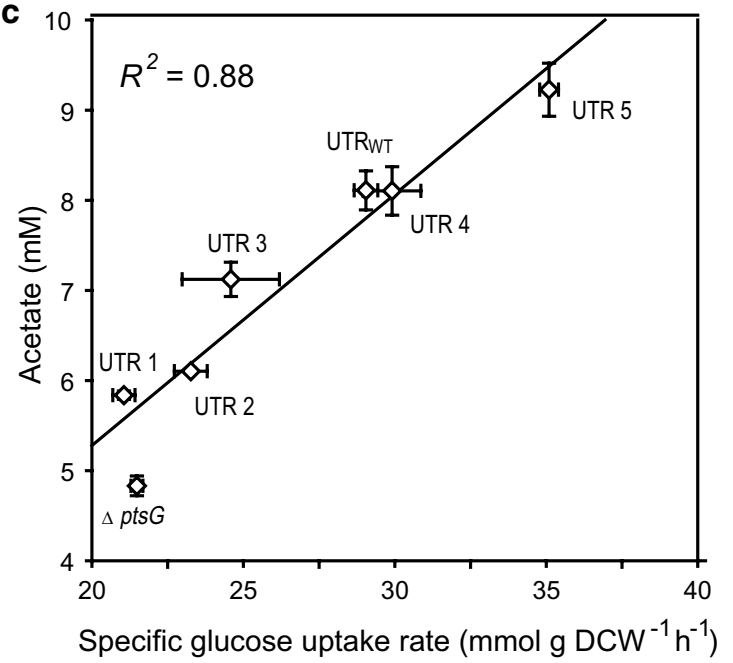

b

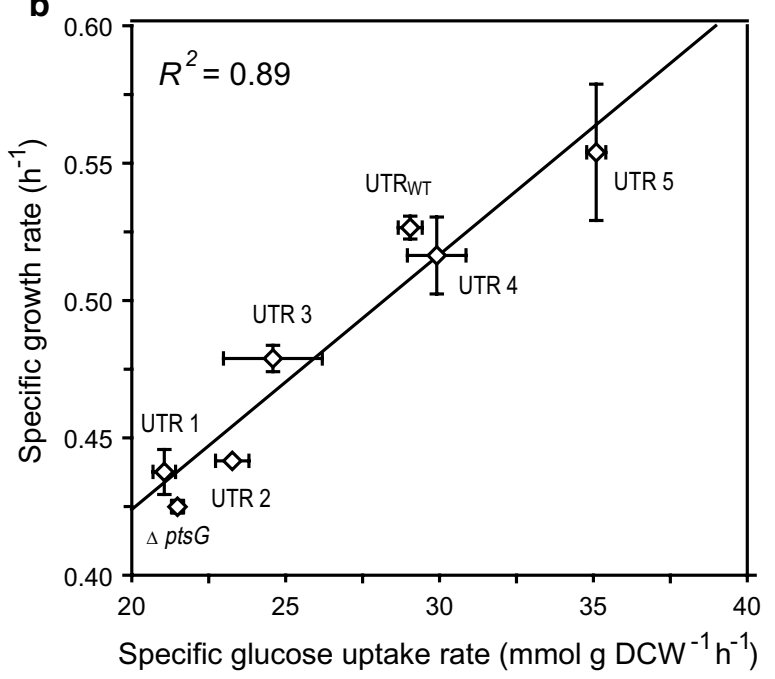

d

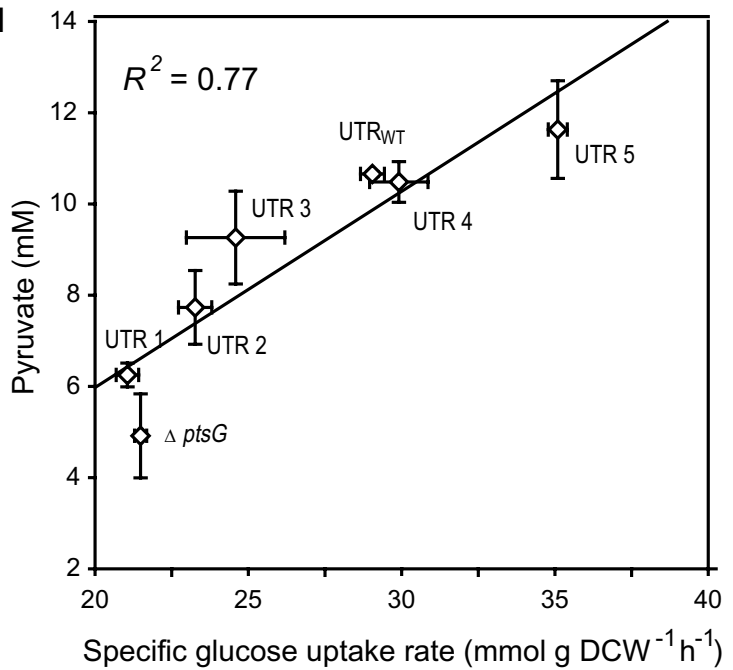

Fig. 3 Physiological comparison among pts G variants of wild-type E. coli W3110. a Schematic metabolic pathway for wild-type E. coli. The dotted red and blue arrows indicate glycolytic flux control and the corresponding change in flux for two natural metabolites, respectively. b Specific growth rate, $\mathbf{c}$ acetate, and $\mathbf{d}$ pyruvate are represented as a function of the specific glucose uptake rate. The specific glucose uptake rate was determined as growth rate divided by biomass yield during exponential growth as previously described [33]. One $\mathrm{OD}_{600}$ unit corresponds to $0.25 \mathrm{~g}$ dry cell weight $(\mathrm{DCW}) / \mathrm{L}$. The error bars indicate standard deviations of measurements from two independent cultures

preliminary rate-determining step, even in the presence of complex regulatory mechanisms for other glycolytic enzymes [14].

\section{Leak-free pathway engineering to improve $n$-butanol cell factory yield}

The $n$-butanol synthetic pathway was selected as an example in which glycolytic activity was higher than product formation under anaerobic condition. Previously, many studies have attempted to optimize the $n$-butanol production pathway, but substantial levels of pyruvate accumulated as a by-product in the medium, indicating the $n$-butanol synthesis pathways are still inefficient $[3,37,38]$. Therefore, in this case, tuning down of glycolytic flux is an effective way to minimize wasteful pyruvate production, which decreases yield (Fig. 4a).

We used an approach that we termed leak-free pathway engineering to improve the yield of the $n$-butanol cell factory. Seven ptsG UTR variants, including the native sequence (JHL 178-183), were engineered using n-butanol-producing E. coli JHL 59 ( $\triangle a t o \mathrm{DA} \triangle a d h E$ $\Delta l d h \mathrm{~A} \quad \Delta p a a \mathrm{FGH} \quad \Delta$ frdABCD $\Delta p t a \mathrm{P}_{a t o \mathrm{~B}}: \mathrm{BBa} \_23100$ $\mathrm{P}_{l p d}: \mathrm{BBa} \_J 23100$ lpd(G1060A) $\left.\mathrm{P}_{\text {aceEF }}: \mathrm{BBa} \_J 23100\right)$ as the parental strain [3]. After a 24-h fermentation period, each variant showed different physiological results in terms of the accumulation of biomass, $n$-butanol, and 
a

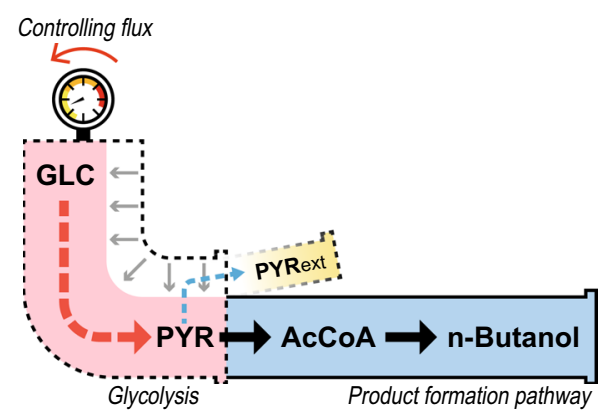

C

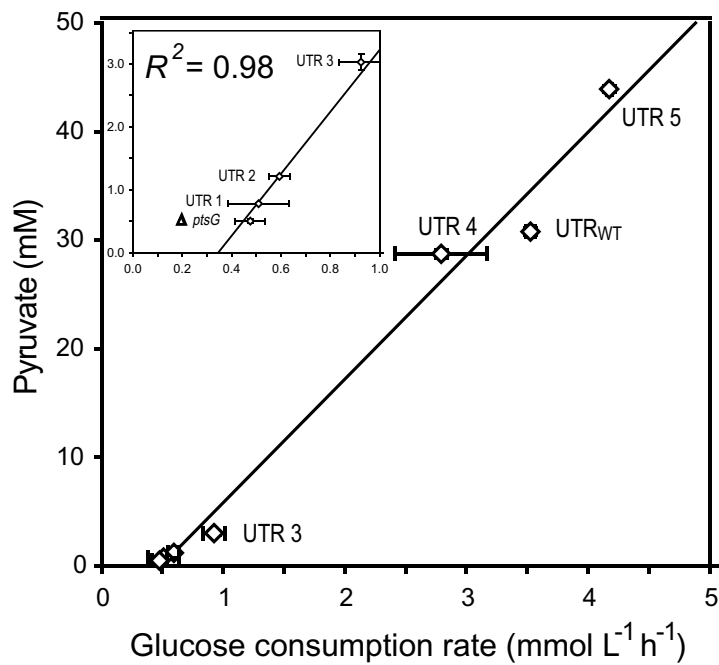

b

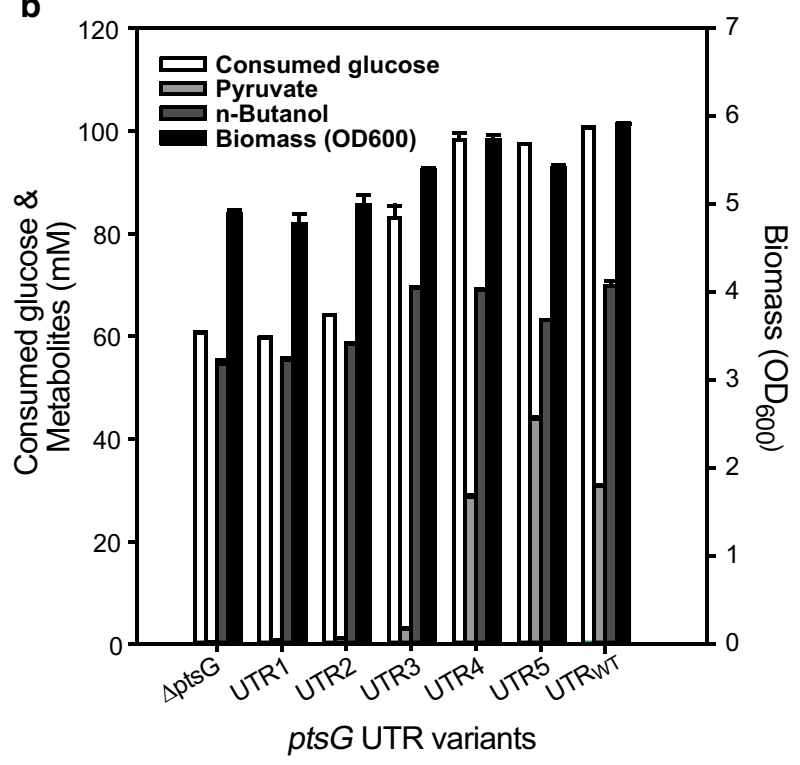

d

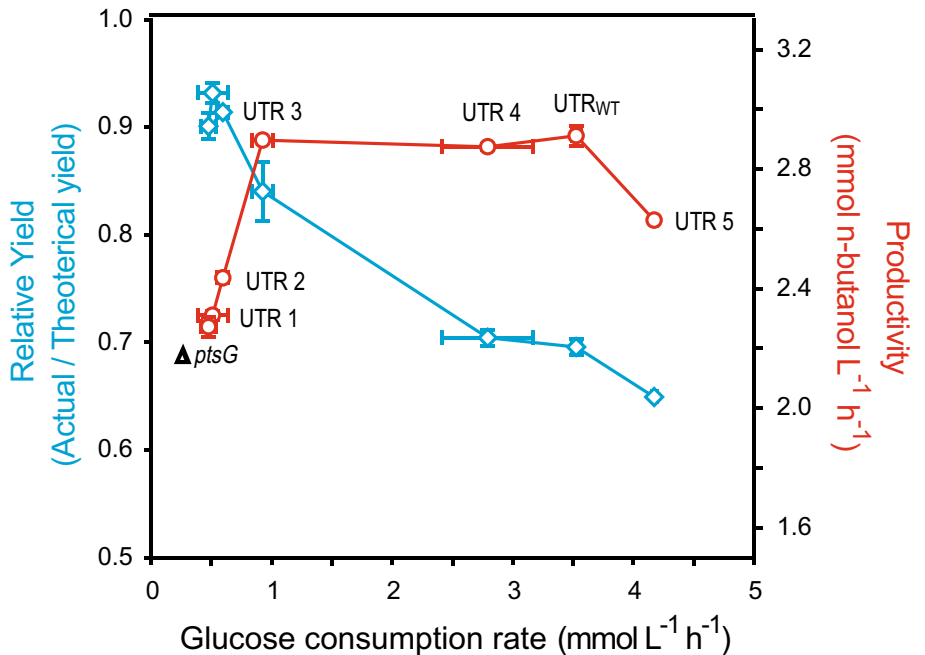

Fig. 4 Tuning the glucose uptake rate to improve the yield of $n$-butanol. a Schematic metabolic pathway for $n$-butanol fermentation. The dotted black outer lines and red and blue arrows indicate controlled glycolytic flux and the corresponding flux change for pyruvate, respectively. b Result of fermentation for pts G UTR variants (Biomass, consumed glucose, $n$-butanol, and pyruvate). c Leaking of pyruvate as a function of the glucose consumption rate. $\mathbf{d}$ Relative yield (blue) and productivity (red) of $n$-butanol depending on the glucose consumption rate. Relative yield represents the ratio compared to the theoretical maximum yield. From left, the open circle represents $\triangle p t s G$, UTR1, UTR2, UTR3, UTR4, UTR were obtained after fermentation for $24 \mathrm{~h}$ in TB medium. It should be noted that the components in the TB medium also contributed to biomass yield; therefore, glycolytic flux was represented as the glucose consumption rate during the initial exponential phase, rather than the specific glucose uptake rate. The error bars indicate standard deviations of measurements from two independent cultures

pyruvate as well as glucose consumption (Fig. 4b). Along with the decrease in the glucose consumption rate, $99 \%$ of pyruvate secretion was successfully eliminated, from $43.92 \mathrm{mM}$ in UTR5 (JHL179) to $0.50 \mathrm{mM}$ in $\Delta p t s G$ (JHL184), by glycolytic flux modulation, and there was a strong correlation between pyruvate secretion and the glucose consumption rate $\left(R^{2}=0.98\right)$ (Fig. 4c). The final titer of $n$-butanol decreased from $69.88 \mathrm{mM}\left(\mathrm{UTR}_{\mathrm{WT}}\right.$, JHL178) to $54.54 \mathrm{mM}$ ( $\Delta p t s G$, JHL184) (Fig. 4b). In addition, the specific growth rate showed strong correlations between the glucose consumption rate $\left(R^{2}=0.93\right)$ and the specific glucose uptake rate $\left(R^{2}=0.94\right)$, even in rich TB medium (Additional file 1: Figures S1, S2, respectively). These results indicate that controlling the ptsG 
expression level through UTR engineering could successfully modulate the glycolytic flux of the engineered strain, even under anaerobic conditions.

To evaluate cellular performance in $n$-butanol production, yield and productivity were examined (Fig. 4d). Notably, $n$-butanol yield increased as glycolytic flux decreased, which was attributed to a reduction in pyruvate leakage (Fig. 4c), but only slight changes in productivity were observed. This clearly shows that reducing glycolytic flux by modifying the glucose uptake rate had a greater influence on pyruvate secretion than $n$-butanol production. Among the tested variants, the JHL181 strain with the UTR3 variant indicated the optimal glycolytic flux for the best trade-off between yield and productivity as it showed $84 \%$ of the theoretical maximum yield by a $20 \%$ improvement ( $0.84 \mathrm{~mol}$ butanol/mol glucose) compared to the parental strain, but exhibited negligible changes in productivity $(2.90 \mathrm{mM}$ butanol L/h for UTR3 vs. $2.91 \mathrm{mM}$ butanol $\mathrm{L} / \mathrm{h}$ for $\mathrm{UTR}_{\mathrm{WT}}$ ) (Fig. $4 \mathrm{~d}$ ). Under the level of UTR3, however, $n$-butanol productivity decreased as a function of the glucose uptake rate, even though the yield increased to $93 \%$ of the theoretical maximum (Please see UTR2 in Fig. 4d). This indicates that glycolytic flux with UTR3 corresponds to the capacity of the engineered $n$-butanol synthesis pathway and glycolytic fluxes below this level can be regarded as the ratelimiting step for the production of $n$-butanol (Fig. 4d). The JHL179 strain with the UTR5 variant, whose rate of glucose uptake was higher $(+18.34 \%)$ than that of the parental strain, showed substantial reductions in yield as well as productivity owing to a significant decrease in $\mathrm{pH}$ resulting from acidic pyruvate accumulation $(+42.65 \%$ compared to UTR $\left.\mathrm{WT}_{\mathrm{W}} ; \mathrm{Fig} .4 \mathrm{c}\right)$, which negatively affected glucose consumption (Fig. 4b). Taken together, our results demonstrate that yield can be maximized while maintaining maximum productivity simply by optimizing the glycolytic flux according to the capacity of the product formation pathways via fine-control of ptsG.

\section{Improvement in productivity by enhanced glycolytic activity through UTR engineering of $p t s G$}

In general, product yield can be maximized via the deletion of pathways for unnecessary by-product formation, but increasing productivity beyond this maximized yield is challenging [7]. Nevertheless, further increases in productivity, while maintaining the maximum yield can be expected by enhancing the glycolytic flux if the capacity of the product formation pathway is higher than the natural glycolytic activity.

To verify this, previously engineered E. coli strains for the production of butyrate [4] and 2,3-butanediol [5] were exploited as model systems; their product yields were close to the theoretical maximum due to the elimination of native by-product formation pathways, such as lactate and ethanol. Since butyrate is a fermentative product, energy for biosynthesis is mostly generated by the butyrate production pathway under anaerobic conditions, and the engineered strain JHL265 showed $83.4 \%$ of the theoretical maximum yield (Fig. 5a) [4]. However, the biological conversion rate of 2,3-butanediol from pyruvate could be maximized in the presence of oxygen and therefore a portion of the carbon source should be consumed to generate energy by conversion to carbon dioxide (Fig. 5b) [39].

To enhance glycolytic activity, pts $G$ expression was activated using UTR5 (resulting JHL266). As expected, the amplified glucose consumption rate translated to $7 \%$ higher productivity for butyrate $(1.45 \mathrm{mmol}$ butyrate $\mathrm{L} / \mathrm{h})$ than the parental strain, while the yield was maintained at approximately $83 \%$ of theoretical maximum (Fig. 5c). In the case of 2,3-butanediol production, the productivity of the strain with higher glycolytic activity (UTR5), resulting JHL268, could be improved by $12.45 \%$ compared to the parental strain JHL267 (2.38 mmol 2,3-butanediol $\mathrm{L} / \mathrm{h}$ ), while maintaining the parental maximum yield (approximately $60 \%$ of the theoretical maximum), as shown in Fig. 5d. Our results clearly show that the productivity of biological processes could be improved by amplifying glycolysis per se through UTR engineering of ptsG.

\section{Discussion}

Although the entire pathway from sugar uptake to product formation has to be well-balanced for optimal yield and productivity, research in metabolic engineering has focused on production pathways. Furthermore, controlling glycolytic flux remains a daunting task owing to incomplete knowledge of the mechanisms that regulate glycolysis [14]. While many process control techniques, such as carbon limited fed-batch cultivation, are the standard approaches to control overflow metabolism [40], our approach has the advantage of increasing robustness of biological production by optimizing glycolytic flux at the genetic level.

In this study, we demonstrated the physiological relevance of $p t s G$ to overall glycolytic activity as the simple method for the control of metabolic input. As small RNA SgrS inherently represses the translation of $p t s G$ mRNA by sequestering its ribosome binding site and RNaseE-dependent cleavage through a short base-pairing interaction [21, 22], the glucose transporter encoded by $p t s G$ was modulated using synthetic $5^{\prime}$-UTRs for the fine-control of translation efficiency in addition to the deregulation of SgrS. Although the molecular study for the UTR engineering-mediated mitigation of SgrS regulation should be further investigated, our physiological 
a

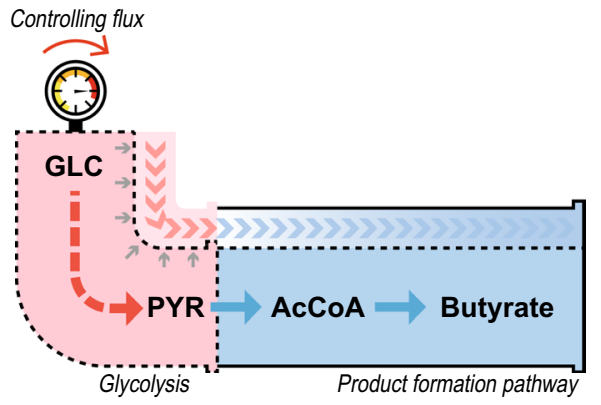

C

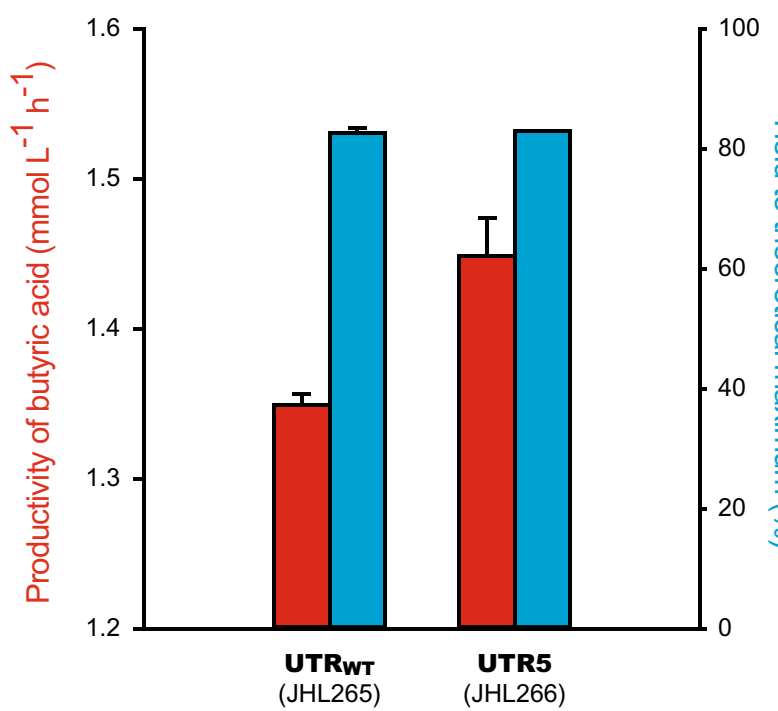

b

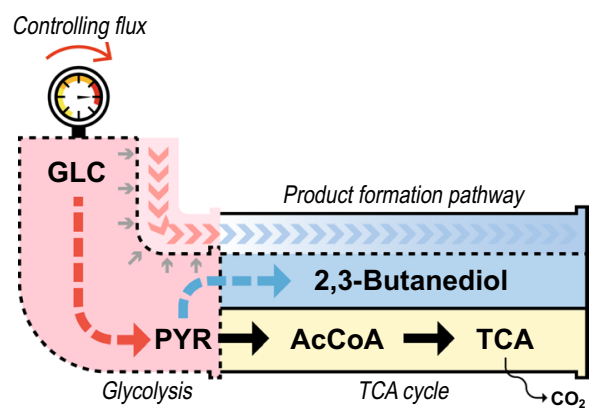

d

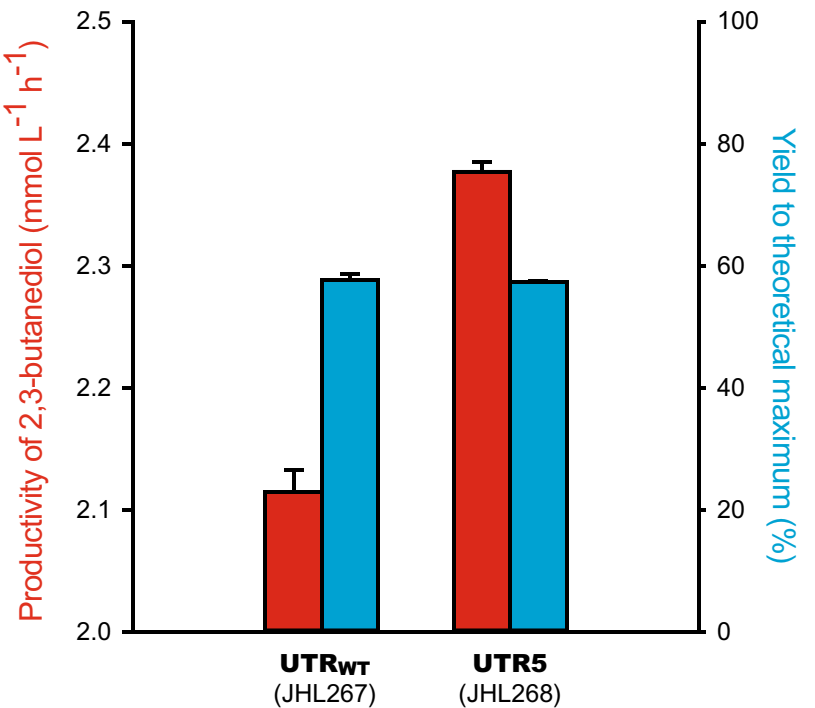

Fig. 5 Comparative analysis of UTR5 to improve the productivity of butyrate and 2,3-butanediol. a Schematic metabolic pathway for the production of $\mathbf{a}$ butyrate and $\mathbf{b}$ 2,3-butanediol. The flux for the TCA cycle was included in the 2,3-butanediol system due to the presence of oxygen. The dotted red and blue arrows indicate controlled glycolytic flux and additional carbon flux from glycolysis to the product-forming pathway, respectively (dotted black outer lines). c The productivity (red) and yield (blue) of butyrate were compared after $24 \mathrm{~h}$ of fermentation in TB medium. $\mathbf{d}$ The productivity (red) and yield (b/ue) of 2,3-butanediol were calculated after $39 \mathrm{~h}$ of cultivation in modified M9 medium. The error bars indicate standard deviations of measurements from two independent cultures

results successfully demonstrate the ability to control the glycolytic flux through ptsG as shown in Fig. 3. Moreover, the redesign of native UTR for allowed an increase in glycolytic flux by $20.8 \%$ compared to the wild type, even though none of the overexpressed glycolytic enzymes increased glycolytic activity in previous studies [41-43]. Since native glycolytic activity is often not sufficient for non-native product formation pathways and therefore increased glycolytic activity is necessary to maximize the rate of product formation for industrial applications, the observation that pts $G$ might be a preliminary rate-determining step in glycolysis is also intriguing.

Using these findings, the optimal glycolytic flux was explored with respect to the capacity of the $n$-butanol, butyrate, and 2,3-butanediol synthesis pathways to improve cellular performance. Interestingly, the yield of $n$-butanol increased to $93 \%$ of the theoretical maximum due to a reduction in pyruvate secretion in accordance with tuning down of the glycolytic flux. Conversely, enhanced productivity was observed for the production of butyrate and 2,3-butanediol by activating the expression level of ptsG (via UTR5). Collectively, these results clearly indicate that optimization of the glycolytic flux enables additional improvements in both yield and productivity of cell factories, beyond optimization of the product formation pathway.

The concept of optimizing glycolytic flux is also important to the microbial production of various chemicals and fuels from cost-effective feedstock, such as glycerol [44] and galactose [45] and our strategy can be applied to explore optimal glycolytic flux depending on the capacity of product formation pathway via fine-control of glycerol 
transporter (encoded by glpF) [46] and galactose transporter (encoded by galP) [47], respectively. Ultimately, as summarized in Fig. 6, balanced pathway amplification of both glycolytic flux and product-forming pathways are highly desirable for the design of economically feasible microbial cell factories in the bio-based chemical industry.

\section{Conclusions}

In this study, we examined the metabolic imbalance between glycolysis and product formation pathways using recombinant Escherichia coli strains producing n-butanol, butyrate, or 2,3-butanediol as model cell factories. Initially, the glucose uptake rate of wild-type $E$. coli was fine-tuned using synthetic UTRs of ptsG to modulate the overall glycolytic fluxes, which were assessed by physiological parameters, i.e., specific growth rate and the accumulation of acetate and pyruvate as natural by-products. Moving forward, glycolytic flux was rebalanced via the control of $p t s G$ depending on the efficiency of product formation pathways with lower (n-butanol) and higher (butyrate and 2,3-butanediol) product formation capacities compared to the wild-type glycolytic flux. For the production of $n$-butanol, glycolytic flux successfully tuned down to minimize by-product formation,

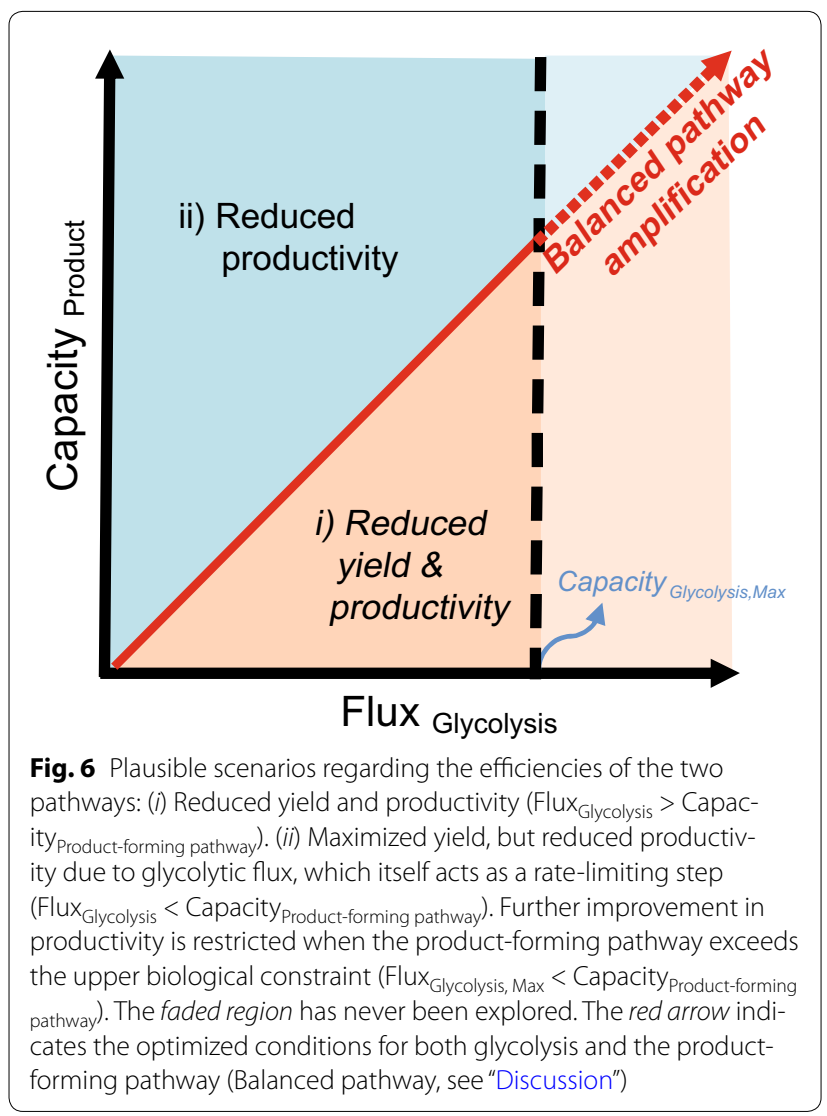

while maintaining productivity, which we termed leakfree pathway engineering. Conversely, butyrate and 2,3-butanediol production rates were increased using a UTR variant of $p t s G$ with higher glycolytic flux than that of the wild type. These results demonstrate the simple method to control glycolytic flux for the design of optimal cell factories in the fields of metabolic engineering and synthetic biology.

\section{Additional file}

Additional file 1. Additional figures, tables and reference.

\section{Abbreviations}

DCW: dry cell weight; OD: optical density; PEP: phosphoenolpyruvate; PTS: phosphotransferase system; TCA: tricarboxylic acid; UTR: untranslated region.

\section{Authors' contributions}

$J \mathrm{HL}$ and GYJ conceived of and designed the study, performed the experiments, analyzed the data, and wrote the manuscript. Both authors read and approved the final manuscript.

\section{Author details}

${ }^{1}$ Department of Chemical Engineering, Pohang University of Science and Technology, 77 Cheongam-Ro, Nam-Gu, Pohang, Gyeongbuk 37673, Korea. ${ }^{2}$ School of Interdisciplinary Bioscience and Bioengineering, Pohang University of Science and Technology, 77 Cheongam-Ro, Nam-Gu, Pohang, Gyeongbuk 37673, Korea.

\section{Acknowledgements}

We are grateful to Dr. Min-Kyu Oh (Korea University) for the generous gift of the plasmid pZSbudABC.

\section{Competing interests}

The authors declare that they have no competing interests.

\section{Availability of supporting data}

All data generated or analyzed during this study are included in this published article and its Additional information file.

\section{Funding}

This research was supported by grants from the Advanced Biomass R\&D Center (ABC) of Global Frontier Project (ABC-2015M3A6A2066119) and C1 Gas Refinery Program (NRF-2016M3D3A1A01913237) through the National Research Foundation of Korea (NRF) funded by the Ministry of Science, ICT and Future Planning, Korea.

\section{Publisher's Note}

Springer Nature remains neutral with regard to jurisdictional claims in published maps and institutional affiliations.

Received: 22 November 2016 Accepted: 13 June 2017

Published online: 21 June 2017

\section{References}

1. Curran KA, Alper HS. Expanding the chemical palate of cells by combining systems biology and metabolic engineering. Metab Eng. 2012;14(4):289-97.

2. Keasling JD. Manufacturing molecules through metabolic engineering. Science. 2010;330(6009):1355-8. 
3. Lim JH, Seo SW, Kim SY, Jung GY. Model-driven rebalancing of the intracellular redox state for optimization of a heterologous $n$-butanol pathway in Escherichia coli. Metab Eng. 2013;20:49-55.

4. Lim JH, Seo SW, Kim SY, Jung GY. Refactoring redox cofactor regeneration for high-yield biocatalysis of glucose to butyric acid in Escherichia coli. Bioresour Technol. 2013;135:568-73.

5. Mazumdar S, Lee J, Oh MK. Microbial production of 2,3-butanediol from seaweed hydrolysate using metabolically engineered Escherichia coli. Bioresour Technol. 2013;136:329-36.

6. Wang BL, Ghaderi A, Zhou H, Agresti J, Weitz DA, Fink GR, et al. Microfluidic high-throughput culturing of single cells for selection based on extracellular metabolite production or consumption. Nat Biotechnol. 2014;32(5):473-8.

7. Van Dien S. From the first drop to the first truckload: commercialization of microbial processes for renewable chemicals. Curr Opin Biotech. 2013;24(6):1061-8.

8. Xu P, Ranganathan S, Fowler ZL, Maranas CD, Koffas MA. Genome-scale metabolic network modeling results in minimal interventions that cooperatively force carbon flux towards malonyl-CoA. Metab Eng. 2011;13(5):578-87.

9. Bogorad IW, Lin TS, Liao JC. Synthetic non-oxidative glycolysis enables complete carbon conservation. Nature. 2013;502(7473):693-7.

10. Farmer WR, Liao JC. Reduction of aerobic acetate production by Escherichia coli. Appl Environ Microb. 1997;63(8):3205-10.

11. Chang DE, Shin S, Rhee JS, Pan JG. Acetate metabolism in a pta mutant of Escherichia coli W3110: importance of maintaining acetyl coenzyme a flux for growth and survival. J Bacteriol. 1999;181(21):6656-63.

12. Xu P, Gu Q, Wang WY, Wong L, Bower AGW, Collins CH, et al. Modular optimization of multi-gene pathways for fatty acids production in E. coli. Nat Commun. 2013;4:1409.

13. Jones JA, Toparlak OD, Koffas MA. Metabolic pathway balancing and its role in the production of biofuels and chemicals. Curr Opin Biotech. 2015:33:52-9.

14. Wadler CS, Vanderpool CK. A dual function for a bacterial small RNA: SgrS performs base pairing-dependent regulation and encodes a functional polypeptide. Proc Natl Acad Sci USA. 2007;104(51):20454-9.

15. Bongaerts J, Kramer M, Muller U, Raeven L, Wubbolts M. Metabolic engineering for microbial production of aromatic amino acids and derived compounds. Metab Eng. 2001;3(4):289-300.

16. Berry A. Improving production of aromatic compounds in Escherichia coli by metabolic engineering. Trends Biotechnol. 1996;14(7):250-6.

17. Solomon KV, Sanders TM, Prather KLJ. A dynamic metabolite valve for the control of central carbon metabolism. Metab Eng. 2012;14(6):661-71.

18. Solomon KV, Moon TS, Ma B, Sanders TM, Prather KL. Tuning primary metabolism for heterologous pathway productivity. ACS Synth Biol. 2013:2(3):126-35.

19. Gosset G. Improvement of Escherichia coli production strains by modification of the phosphoenolpyruvate: sugar phosphotransferase system. Microb Cell Fact. 2005:4:14.

20. Deutscher J, Francke C, Postma PW. How phosphotransferase systemrelated protein phosphorylation regulates carbohydrate metabolism in bacteria. Microbiol Mol Biol Rev. 2006;70(4):939-1031.

21. Morita T, El-Kazzaz W, Tanaka Y, Inada T, Aiba H. Accumulation of glucose 6-phosphate or fructose 6-phosphate is responsible for destabilization of glucose transporter mRNA in Escherichia coli. J Biol Chem. 2003;278(18):15608-14.

22. Kimata K, Tanaka Y, Inada T, Aiba H. Expression of the glucose transporter gene, ptsG, is regulated at the mRNA degradation step in response to glycolytic flux in Escherichia coli. EMBO J. 2001;20(13):3587-95.

23. Morita T, Maki K, Aiba H. RNase E-based ribonucleoprotein complexes: mechanical basis of mRNA destabilization mediated by bacterial noncoding RNAs. Genes Dev. 2005;19(18):2176-86

24. Vanderpool CK, Gottesman S. Involvement of a novel transcriptional activator and small RNA in post-transcriptional regulation of the glucose phosphoenolpyruvate phosphotransferase system. Mol Microbiol. 2004:54(4):1076-89.

25. Kawamoto H, Morita T, Shimizu A, Inada T, Aiba H. Implication of membrane localization of target mRNA in the action of a small RNA mechanism of post-transcriptional regulation of glucose transporter in Escherichia coli. Genes Dev. 2005;19(3):328-38.

26. Maki K, Morita T, Otaka H, Aiba H. A minimal base-pairing region of a bacterial small RNA SgrS required for translational repression of ptsG mRNA. Mol Microbiol. 2010:76(3):782-92.

27. Seo SW, Yang J, Min BE, Jang S, Lim JH, Lim HG, et al. Synthetic biology: tools to design microbes for the production of chemicals and fuels. Biotechnol Adv. 2013;31(6):811-7.

28. Datsenko KA, Wanner BL. One-step inactivation of chromosomal genes in Escherichia coli K-12 using PCR products. Proc Natl Acad Sci USA. 2000:97(12):6640-5.

29. Lim SI, Min BE, Jung GY. Lagging strand-biased initiation of Red recombination by linear double-stranded DNAs. J Mol Biol. 2008;384(5):1098-105.

30. Heermann R, Zeppenfeld T, Jung K. Simple generation of site-directed point mutations in the Escherichia coli chromosome using Red (R)/ET (R) recombination. Microb Cell Fact. 2008;7:14.

31. Seo SW, Yang JS, Kim I, Yang J, Min BE, Kim S, et al. Predictive design of mRNA translation initiation region to control prokaryotic translation efficiency. Metab Eng. 2013:15:67-74.

32. Negrete A, Ng WI, Shiloach J. Glucose uptake regulation in E. coli by the small RNA SgrS: comparative analysis of E. coli K-12 (JM109 and MG1655) and E. coli B (BL21). Microb Cell Fact. 2010;9:75.

33. Varma A, Palsson BO. Stoichiometric flux balance models quantitatively predict growth and metabolic by-product secretion in wild-type Escherichia coli W3110. Appl Environ Microb. 1994;60(10):3724-31.

34. Jung J, Lim JH, Kim SY, Im DK, Seok JY, Lee SV, et al. Precise precursor rebalancing for isoprenoids production by fine control of gapA expression in Escherichia coli. Metab Eng. 2016;38:401-8.

35. Kayser A, Weber J, Hecht V, Rinas U. Metabolic flux analysis of Escherichia coli in glucose-limited continuous culture. I. Growth-rate-dependent metabolic efficiency at steady state. Microbiology. 2005;151(Pt 3):693-706.

36. Wolfe AJ. The acetate switch. Microbiol Mol Biol Rev. 2005;69(1):12-50.

37. Shen CR, Lan El, Dekishima Y, Baez A, Cho KM, Liao JC. Driving forces enable high-titer anaerobic 1-butanol synthesis in Escherichia coli. Appl Environ Microb. 2011;77(9):2905-15.

38. Nielsen DR, Leonard E, Yoon SH, Tseng HC, Yuan C, Prather KLJ. Engineering alternative butanol production platforms in heterologous bacteria. Metab Eng. 2009:11(4-5):262-73.

39. Ji XJ, Huang H, Ouyang PK. Microbial 2,3-butanediol production: a stateof-the-art review. Biotechnol Adv. 2011;29(3):351-64.

40. Eiteman MA, Altman E. Overcoming acetate in Escherichia coli recombinant protein fermentations. Trends Biotechnol. 2006:24(11):530-6.

41. Koebmann BJ, Westerhoff HV, Snoep JL, Nilsson D, Jensen PR. The glycoIytic flux in Escherichia coli is controlled by the demand for ATP. J Bacteriol. 2002;184(14):3909-16.

42. Ruyter GJ, Postma PW, van Dam K. Control of glucose metabolism by enzyme IIGlc of the phosphoenolpyruvate-dependent phosphotransferase system in Escherichia coli. J Bacteriol. 1991;173(19):6184-91.

43. Jojima T, Inui M. Engineering the glycolytic pathway: a potentia approach for improvement of biocatalyst performance. Bioengineered. 2015:6(6):328-34

44. Lim HG, Noh MH, Jeong JH, Park S, Jung GY. Optimum rebalancing of the 3-hydroxypropionic acid production pathway from glycerol in Escherichia coli. Acs Synth Biol. 2016;5(11):1247-55.

45. Lim HG, Lim JH, Jung GY. Modular design of metabolic network for robust production of $n$-butanol from galactose-glucose mixtures. Biotechnol Biofuels. 2015:8:137.

46. Murarka A, Dharmadi Y, Yazdani SS, Gonzalez R. Fermentative utilization of glycerol by Escherichia coli and its implications for the production of fuels and chemicals. Appl Environ Microbiol. 2008;74(4):1124-35.

47. Lim HG, Seo SW, Jung GY. Engineered Escherichia coli for simultaneous utilization of galactose and glucose. Bioresour Technol. 2013;135:564-7.

48. Kawamoto H, Koide Y, Morita T, Aiba H. Base-pairing requirement for RNA silencing by a bacterial small RNA and acceleration of duplex formation by Hfq. Mol Microbiol. 2006;61(4):1013-22. 